\title{
A Maximum Likelihood Digital Receiver Using Coordinate Ascent and the Discrete Wavelet Transform
}

\author{
Ilan Sharfer, Member, IEEE, and Alfred O. Hero, III, Fellow, IEEE
}

\begin{abstract}
In this paper, a maximum likelihood (ML) method is presented for joint estimation of amplitude, phase, time delay, and data symbols in a single-user direct-sequence spreadspectrum communication system. Since maximization of the likelihood function is analytically intractable, a novel coordinate ascent algorithm is used to obtain sequential updates of the data symbols and all unknown nuisance parameters. The novelty of the algorithm is due to the use of a multiresolution expansion of the received signal and the use of polynomial rooting in the complex plane in place of a line search over the signal delay parameter. The multiresolution structure of the algorithm is exploited to reduce sensitivity to impulsive noise via wavelet thresholding. Computer simulations of the single-user system show that the algorithm has fast convergence, and comparison with theoretical lower bounds establishes that the algorithm achieves nearly optimal error performance.
\end{abstract}

Index Terms-Bit synchronization, carrier phase recovery, impulsive noise mitigation, sequence estimation, wireless communications.

\section{INTRODUCTION}

$\mathbf{T}$ HE NEW technologies of multiuser wireless communication systems, mobile radio, and personal communication networks require advanced signal processing methods for improved efficiency and reliability. The growing computing power and shrinking cost of digital signal processing (DSP) technology makes sophisticated signal processing algorithms both practical and affordable. Wireless communication systems must frequently operate in harsh environmental conditions that adversely effect the transmitted signal through such phenomena as time-varying channel response, multipath fading, multiaccess and cochannel interference, non-Gaussian noise, and loss of synchronization. A parametric model of the received signal typically includes unknown amplitude, phase, and time delay, which should be accurately estimated to ensure near optimal decoding of the data symbols. Traditionally, these

Manuscript received July 7, 1997; revised July 7, 1998. This work was supported in part by the Army Research Office under Grant DAAH04-96-10337. Portions of this work appeared in the Proceedings of the 1997 Workshop on Signal Processing Advances in Communications, Paris, France, and in the Proceedings of the 1995 International Conference on Acoustics, Speech, and Signal Processing, Detroit, MI. The associate editor coordinating the review of this paper and approving it for publication was Prof. Georgios B. Giannakis.

I. Sharfer was with the Department of Electrical Engineering and Computer Science, University of Michigan, Ann Arbor, MI 48109-2122 USA. He is now with Orckit Communications, Tel-Aviv, Israel.

A. O. Hero, III is with the Department of Electrical Engineering and Computer Science, University of Michigan, Ann Arbor, MI 48109-2122 USA (e-mail: hero@eecs.umich.edu).

Publisher Item Identifier S 1053-587X(99)01345-8. nuisance parameters are estimated by nonoptimal combination of several subsystems, each specialized to estimating a particular parameter. For example, carrier phase and time delay estimation are frequently done with a phase-locked loop (PLL) and a delay-locked loop (DLL), respectively [24]. The PLL and DLL techniques are equivalent to the optimal maximum likelihood (ML) or maximum a posteriori probability (MAP) estimators under the assumptions that the data symbols are known and that either the symbol timing is known (PLL) or the carrier phase is known (DLL). However, the overall estimator is suboptimal, and it may unnecessarily reduce the system's operational threshold. Therefore, an optimal receiver that jointly estimates the nuisance parameters as well as the data symbols is sought in order to achieve better performance.

A considerable amount of research has gone into improving the performance of the basic PLL and DLL synchronization techniques, e.g., by using decision feedback in a data-aided loop (DAL) [2], [23], a decision-directed receiver [22], or by deriving optimal nondata-aided estimation structures [27]. More recently, digital implementations of pseudo-noise (PN) code tracking algorithms using the extended Kalman filter have been proposed [21]. The problem of maximum likelihood sequence estimation for the intersymbol interference (ISI) channel has been treated in [15] using a whitened matched filter followed by a Viterbi decoding algorithm. Most of these estimation techniques critically depend on the Gaussian noise assumption; therefore, they become ineffective when the interference contains a dominant impulsive noise component. The joint parameter estimation and data demodulation problem becomes even more difficult in a multiuser communication system such as the code division multiple access (CDMA) system, which provided the motivation for the work reported in this paper.

This paper presents a novel joint ML estimator of the unknown parameters in the context of a single user receiver. First an ML estimation algorithm for the additive white Gaussian noise (AWGN) channel is developed, which is subsequently robustified for the non-Gaussian impulsive noise channel. The ML estimator yields an optimal receiver that generates estimates of the nuisance parameters that are asymptotically uniform minimum variance unbiased (UMVUE) in the sense of achieving the CR bound in the limit of large number of observations. The single-user ML algorithm has been adapted to multiuser channels, but due to space limitations, this is treated elsewhere [36], [37]. In addition, while such extensions 
have not yet been fully explored, it is probably straightforward to apply the approach of this paper to the case of fading and ISI channels.

It is well known that direct maximization of the joint likelihood function is analytically intractable due to the unknown delay parameter. Several suboptimal schemes for approximating the joint maximum likelihood sequence and synchronization parameters have been recently proposed [5], [33], [34]. Another approach is iterative ML estimation using steepest descent, Newton-Raphson, or the expectation-maximization (EM) algorithm, which have been used in similar problems involving superposition of signals [3], [4], [11], [12], [16]. These algorithms are limited to block processing. All the unknown parameters and, in particular, all the data symbols within a block must be updated simultaneously. Furthermore, many of these algorithms are known to suffer from divergence or very slow convergence. This results in a large processing delay that can only be compensated by increasing processor speed and receiver power consumption. For the case of known signal delay (synchronous case), the Viterbi algorithm performs maximum likelihood sequential decoding in a single pass over the symbols sequence [15]. However, the single-pass Viterbi algorithm cannot be implemented as an ML decoder for the asynchronous case. The iterative ML algorithm proposed in this paper uses coordinate ascent maximization to jointly estimate amplitude, phase, time delay, and data symbols in a sequential manner. Furthermore, by performing the parameter updates on the coefficients of a multiresolution decomposition of the received signal, a single pass algorithm is obtained.

Coordinate ascent is an iterative maximization technique that cycles over groups (or coordinates) of parameters and guarantees that the likelihood is increased at each iteration. It is related to a class of EM algorithms known as space-alternating generalized EM (SAGE) algorithms introduced in [13] and applied to the synchronous multiuser synchronous CDMA detection problem in [30]. Standard EM algorithms have been applied to synchronous sequence estimation in the presence of fading [17] and unknown carrier phase [32]. The algorithm in this paper handles both unknown carrier phase and unknown bit synchronization and is equivalent to a SAGE algorithm for a special choice of hidden data sets (complete data) that are defined as one-to-one transformations of the observations (incomplete data). As a result, it can be shown that this coordinate ascent algorithm is a SAGE algorithm that has the fastest possible asymptotic convergence for our particular parameter cycling strategy. Since, for signal superposition problems such as the communications application in this paper, the asymptotic convergence speed of SAGE is significantly faster than standard EM [13], this coordinate ascent algorithm does not suffer from the slow convergence inherent in EM algorithms.

As in [19] and [28], a well-adapted orthogonal signal representation of the measurements is used as a way to concentrate parameter and symbol information into a low number of coefficients. These representations cover the conventional cardinal series basis, the time-limited sinusoidal basis functions [20], the Walsh basis, the exponential basis [19], the Karhunen-Loeve basis, and the Slepian basis [38].
The orthogonal representation is exploited by re-expressing the likelihood function as a polynomial in a complex variable, which is then used to solve for the unknown delay by fast rootfinding methods. Next, this is specialized to the Daubechies wavelet basis [7], [41] to obtain a multiresolution data recursive implementation of the coordinate ascent ML algorithm. Wavelets have the advantage of time and scale localization, which make them well suited for recursive parameter update algorithms for which each parameter affects the signal at different times and scales. In the present application, the amplitude and phase parameters are coarse scale parameters in the sense that changes in these parameters affects the signal by a global scale factor over time. On the other hand, the data symbols are finer scale parameters since they only affect the signal locally in time. The multiresolution property is important for the implementation of the coordinate ascent algorithm as it separately encodes coarse scale parameters, e.g., signal amplitude and phase, and finer scale parameters, e.g., data symbols and timing, into different sets of wavelet coefficients.

Wavelet-based signal processing techniques have recently seen very rapid growth in related areas. They have demonstrated their usefulness in seismology, image processing, and data compression as well as in many other applications. Wavelet bases enjoy a very strong optimality property for general inverse problems in that their use can achieve accurate and parsimonious representation of the input signal and simultaneously diagonalize the channel [10]. In addition, wavelet and other orthogonal modulation schemes have been suggested as a means of attaining reliable communication over multiple access, fading, and ISI channels [43]-[45]. While such modulation schemes are not required for implementation of the ML receiver presented here, the receiver is naturally adapted to them.

Wavelet domain signal processing can also be very effective in suppression of impulsive noise that exists in wireless and optical communications as well as wireline communications such as digital subscriber lines and cable TV plants. This type of temporally localized noise contaminates a significant portion of the usable transmission spectrum. The optimum receiver for impulsive noise is hard to derive, whereras narrowband noise rejection techniques are not effective. Although the influence of impulsive noise can be reduced by the use of additional coding [29], this comes at a cost of reduced data rate and/or increased delay. The temporal localization of the wavelet basis can be used to detect and reject impulsive events using a simple thresholding algorithm [1], [10]. The reasoning is that only the largest wavelet coefficients are likely to be significantly corrupted by the noise impulses. It will be shown how this thresholding technique can be easily incorporated within the general framework of iterative ML sequence estimation.

The paper is organized as follows. In Section II, the single user observation model is defined. In Section III, the form of the likelihood function is given, and simplifications of the line search step are discussed, which result in a coordinate ascent algorithm with increasing complexity as a function of the block size. In Section IV, a fixed complexity algorithm is 
obtained by using wavelet representations. In Section V, the fixed complexity algorithm is robustified against impulsive noise interference via wavelet thresholding. In Section VI, simulation results are presented, and Section VII concludes the paper.

\section{OBSERVATION Model}

Consider the single-user complex baseband model for the received signal

$$
Y(t)=s(t)+u(t) \quad 0 \leq t \leq T
$$

where $s(t)$ is an attenuated and delayed version of the data modulated signal transmitted by the user

$$
s(t)=a \sum_{n=0}^{N-1} b_{n} p\left(t-n T_{b}-d\right) .
$$

Note that $s(t)$ is a superposition of delayed and scaled versions of the signaling waveform $p(t)$, which is assumed to be a known PN code. For simplicity, it is assumed that the period of the PN code is equal to the data symbol time interval $T_{b}$. Note that it is not necessary to assume that the support of $p(t)$ is the same as the symbol interval-this property would be critical for extension of the algorithm to ISI channels. The noise $u(t)$ will be assumed to be a complex white Gaussian process with power spectral density $N_{0} / 2$.

The unknown parameters in the model (2) are the complex gain $a \in\{R \exp (i \phi): R>0,0 \geq \phi<\pi\}$, the data symbols $b_{n} \in\{ \pm 1\}$ for $n=0, \cdots, N-1$, and the time delay $d$. It is assumed that $d$ is contained in the interval $\left[-T_{d} / 2, T_{d} / 2\right]$ for some positive $T_{d} \ll T$. The $n$ index set notation $I_{N}=\{0, \cdots, N-1\}$ will be used. For simplicity, the data symbols $b_{n}$ in (2) are assumed to be in BPSK modulation format, but with minor modifications, the cases of $M$-ary PSK, $\mathrm{PAM}$, and QAM can be treated. The restriction that $a$ lies in the upper half of the complex plane is required in order to remove the $180^{\circ}$ phase ambiguity and to enable coherent demodulation of the transmitted symbols. There are several techniques for resolving phase ambiguity at the expense of a small decrease in spectral efficiency [24, ch. 2]. Alternatively, DPSK modulation can be used to encode the source into phase changes rather than absolute phase of the transmitted signal.

\section{JOINT MAXIMUM LIKELIHOOD ESTIMATION}

\section{A. Direct Maximization of Likelihood}

Let $\left\{\psi_{k}\right\}_{k=1}^{\infty}$ be a real orthonormal basis for the space of square integrable function $\mathcal{H}$. Relative to this basis, the continuous time observation $Y$ can be converted to the equivalent discrete time observation

$$
Y_{k}=\left\langle Y, \psi_{k}\right\rangle \quad k=1,2, \cdots
$$

where $\langle f, g\rangle$ denotes the inner product $\int f(t) g^{*}(t) d t$. The original observation can be recovered from the discrete time observation through the reconstruction formula

$$
Y(t)=\sum_{k} Y_{k} \psi_{k}(t)
$$

Therefore, the coefficients $\left\{Y_{k}\right\}$ are sufficient statistics for the original continuous time observations $Y$. Given that the observation noise $u(t)$ is a white complex Gaussian process with spectral level $N_{0} / 2$, it follows from (1) and (2) that $\left\{Y_{k}\right\}$ is a set of independent jointly Gaussian complex random variables with mean

$$
E\left\{Y_{k}\right\}=a \sum_{n=0}^{N-1} b_{n} w_{k}(n ; d), \quad k=1,2, \cdots
$$

and variance $N_{0} / 2$, where $w_{k}(n ; d)$ are the projection coefficients of the time function $\left\{p\left(t-n T_{b}-d\right)\right\}_{t \in[0, T]}$,

$$
w_{k}(n ; d)=\int_{-\infty}^{\infty} p\left(t-n T_{b}-d\right) \psi_{k}(t) d t .
$$

In the sequel, the unknown parameters are collectively referred to as the $N+2$ element parameter vector $\theta=$ $\left[a, d, b_{0}, \cdots, b_{N-1}\right]^{T}$. Thus, up to known additive constants, the log-likelihood function can be expressed as

$$
\begin{aligned}
\log p(Y ; \theta)=\frac{2}{N_{0}}[ & a^{*} \sum_{k} Y_{k} \sum_{n=0}^{N-1} b_{n}^{*} w_{k}^{*}(n ; d) \\
& +a \sum_{k} Y_{k}^{*} \sum_{n=0}^{N-1} b_{n} w_{k}(n ; d) \\
& \left.-|a|^{2} \sum_{k}\left|\sum_{n=0}^{N-1} b_{n} w_{k}(n ; d)\right|^{2}\right] .
\end{aligned}
$$

A necessary and sufficient condition for $\hat{\theta}=[\hat{a}, \hat{d}$, $\left.\hat{b}_{0}, \cdots, \hat{b}_{N-1}\right]^{T}$ to jointly maximize the $\log$ likelihood (5) is that three equations be satisfied.

$$
\begin{gathered}
\hat{d}=\arg \max _{d \in\left[-T_{d} / 2, T_{d} / 2\right]} \log p\left(Y ;\left[\hat{a}, d, \hat{b}_{0}, \cdots, \hat{b}_{N-1}\right]\right) \\
\hat{b}_{n}=\arg \max _{b_{n n} \in\{-1,1\}} \log p\left(Y ;\left[\hat{a}, \hat{d}, \hat{b}_{0}, \cdots, b_{n}, \cdots, \hat{b}_{N-1}\right]\right) \\
n=0, \cdots, N-1
\end{gathered}
$$

and

$$
\hat{a}=\frac{\sum_{k} Y_{k} \sum_{n=0}^{N-1} \hat{b}_{n}^{*} w_{k}^{*}(n ; \hat{d})}{\sum_{k} \mid \sum_{n=0}^{N-1} \hat{b}_{n} w_{k}\left(n ;\left.\hat{d}\right|^{2}\right.} .
$$

Note that only one of these equations (8) is explicit. The solution of (7) requires searching over $2^{N}$ possible symbol values $\left\{b_{n} \in\{-1,1\}\right\}_{n=0}^{N-1}$, and the solution of (6) requires performing a line search over $d \in\left[-T_{d} / 2, T_{d} / 2\right]$. The high complexity of these latter solutions makes direct maximization impractical and provides the primary motivation for this work. The first step is to simplify the line search.

\section{B. Line Search via Polynomial Rooting}

First, it will be necessary to transform (5) into a polynomial equation in the complex variable $z=e^{j \omega_{0} d}$ by representing the projection coefficients $w_{k}(n ; d)$ in Fourier series in the delay 
variable $d$. The benefits of this transformation will become clear: A computationally intensive line search for the maximum over $d$ of (5) is eliminated, and the numerical solution can be found by standard polynomial rooting algorithms that are fast and reliable. Suppose that we are interested in the inner product $w_{k}(n ; d)=\left\langle p\left(\cdot-n T_{b}-d, \psi_{k}\right\rangle\right.$ on some interval $d \in\left[-T_{0} / 2, T_{0} / 2\right]$ that contains the a priori time delay uncertainty interval $\left[-T_{d} / 2, T_{d} / 2\right]$. Define the Fourier coefficients

$$
c_{k, m}(n)=\sqrt{T_{0}} \int_{-\infty}^{\infty} v_{T_{0}}(t) w_{k}(n ; t) e^{-j m \omega_{0} t} d t
$$

where

$$
v_{T_{0}}(t)= \begin{cases}1 / T_{0} & |t| \leq T_{0} / 2 \\ 0 & |t|>T_{0} / 2\end{cases}
$$

and $\omega_{0}=2 \pi / T_{0}$. By the convolution property of the Fourier transform

$$
c_{k, m}(n)=\left.\frac{\sqrt{T_{0}}}{2 \pi} W_{k}(n ; \omega) * V_{T_{0}}(\omega)\right|_{\omega=m \omega_{0}}
$$

where $V_{T_{0}}(\omega)=\sin \left(\omega T_{0} / 2\right) /\left(\omega T_{0} / 2\right)$ is the Fourier transform of $v_{T_{0}}(t)$, and $W_{k}(n ; \omega)=\int_{-\infty}^{\infty} w_{k}(n ; \tau) e^{-j \omega \tau} d \tau$ is the Fourier transform of $w_{k}(n ; d)$ with respect to $d$.

The Fourier series expansion of $w_{k}(n ; d)$ over $d \in$ $\left[-T_{0} / 2, T_{0} / 2\right]$ can be written as a function of the complex variable $z$

$$
w_{k}(n ; d)=\sum_{m \in \mathbb{Z}} c_{k, m}(n) z^{m}
$$

with $z=e^{j \omega_{0} d}$. Since $w_{k}(n ; d)$ is a real function of $d$, the Fourier coefficients $c_{k, m}(n)$ are conjugate symmetric in $m$

$$
c_{k, m}(n)=c_{k,-m}^{*}(n) \quad m \in \mathbb{Z}
$$

and $c_{k, 0}(n)$ is real.

With the series representation (11), the log-likelihood (5) can be approximated to arbitrary accuracy by a polynomial in the complex variable $z$. To see this, use conjugate symmetry of the Fourier coefficients to rewrite (5) as the double-sided infinite power series

$$
\log p(Y ; \theta)=\sum_{m \in \mathbb{Z}} q_{m} z^{m}=F(z)+F^{*}\left(z^{*-1}\right)
$$

where $F(z)=\sum_{m=1}^{\infty} q_{m} z^{m}+q_{0} / 2$, and $q_{m}$ is the coefficient of $e^{j m \omega_{0} d}$ in the log likelihood expansion, that is

$$
\begin{gathered}
q_{m}=\frac{2}{N_{0}}\left[-|a|^{2} \sum_{n=0}^{N-1} \sum_{k} \sum_{p \in \mathbb{Z}} c_{k, p}(n) c_{k, m-p}(n)+\right. \\
\left.a \sum_{n=0}^{N-1} b_{n} \cdot \sum_{k} Y_{k}^{*} c_{k, m}(n)+a^{*} \sum_{n=0}^{N-1} b_{n}^{*} \sum_{k} Y_{k} c_{k, m}^{*}(n)\right] .
\end{gathered}
$$

Note that $F(z)$ depends on the parameter values $a$ and $b_{0}, \cdots, b_{N-1}$.

Now, let $\epsilon>0$ be arbitrary, and define $D_{f}$ as the smallest positive integer such that

$$
\frac{1}{N} \sum_{m>D_{f}} E\left[\left|q_{m}\right|^{2}\right]<\epsilon / 2 .
$$

Define the order $D_{f}$ polynomial $\tilde{F}(z)=\sum_{m=1}^{D_{f}} q_{m} z^{m}+q_{0} / 2$. Then

$$
Q(z)=\tilde{F}(z)+\tilde{F}^{*}\left(z^{*-1}\right)
$$

is a polynomial approximation to the log likelihood in the mean square sense

$$
\frac{1}{N} E\left[|\log p(Y ; \theta)-Q(z)|^{2}\right]<\epsilon .
$$

Note that the degree $D_{f}$ of $\tilde{F}(z)$ is finite since the observations have finite power. $D_{f}$ will principally depend on the bandwidth of the signaling waveform $p(t)$, which for PN sequences, is proportional to the number of chips per symbol interval, i.e., the processing gain, since the Fourier coefficients $c_{k, m}(n)$ decay rapidly as $m \omega_{0}$ increases beyond the bandwidth of $p(t)$.

In the sequel, the notation $Q=Q_{a, \underline{b}}$ will be used to clarify the fact that $Q$ is a function of the amplitude $a$ and the symbols $\underline{b}=\left[b_{0}, \cdots, b_{N-1}\right]$. Note that since $Q_{a, b}$ is a smooth function, the maximum $d=d_{\max }$ of $Q_{a, \underline{b}}\left(e^{j \omega_{0} d}\right)$ over $d \in\left[-T_{0} / 2, T_{0} / 2\right]$ will occur within $O(\epsilon)$ of the ML estimate $\hat{d}$, and there is a stationary point at this maximum, i.e., $\left.(\partial / \partial d) Q_{a, b}\left(e^{j \omega_{0} d}\right)\right|_{d=d_{\max }}=0$. The idea is to add a penalty function that will force $Q_{a, \underline{b}}(z)$ to have a stationary point near $z=e^{j \omega_{0} d_{\max }}$ and such that the stationary points can be found by rooting a polynomial. To this aim, define the penalized polynomial

$$
\phi_{a, \underline{b}}(z)=Q_{a, \underline{b}}(z)+\log \Pi(z)
$$

where $\Pi(z)$ is a rational function of the form

$$
\Pi(z)=\frac{G(z) G^{*}\left(z^{*-1}\right)}{H(z) H^{*}\left(z^{*-1}\right)}
$$

where $G(z)$ and $H(z)$ are polynomials of degree $D_{g}$ and $D_{h}$, respectively, over $\mathbb{C}$

$$
G(z)=\sum_{n=0}^{D_{g}} g_{n} z^{n} ; \quad H(z)=\sum_{n=0}^{D_{h}} h_{n} z^{n} .
$$

Now, assume that $\Pi(z)$ is a function that satisfies the following. 1) $\Pi(z)$ is positive and reasonably flat on the semicircle $\left\{z=e^{i \omega}:|\omega| \leq \omega_{0} T_{d} / 2\right\}$. 2) Magnitude of $\Pi(z)$ decays quickly to zero as $z$ deviates from the semi-circle. Then, any stationary points of $\phi_{a, \underline{b}}(z)$ that are on the unit circle will be close to the stationary points of $Q_{a, b}\left(e^{j \omega_{0} d}\right)$ over $d \in\left[-T_{d} / 2, T_{d} / 2\right]$. It follows that the line search step (6) of the joint ML estimator can be approximated by the following steps:

1) Differentiate the function $\phi(z)=\phi_{a, b}(z)$ (13) with respect to $z$. This gives a rational function

$$
\phi^{\prime}(z)=\frac{Q^{\prime}(z) \Pi(z)+\Pi^{\prime}(z)}{\Pi(z)}=A(z) / B(z)
$$

where $A(z)$ is a polynomial of degree $D=2\left(D_{f}+\right.$ $D_{g}+D_{h}$ ).

2) Find the roots of the numerator polynomial $A(z)$ that lie on the unit circle. 
3) Evaluate the objective $\phi(z)$ at each of these roots, and select the root that maximizes the penalized objective (13).

4) The phase of the maximizing root specifies the estimate of $d$.

Since the numerator polynomial of (16) has fixed degree $D$, there is a fixed computational complexity per iteration of the delay estimation step. The penalty function $\Pi(z)$ can be implemented by applying standard filter design methods [31] to the transfer function $G(z) / H(z)$ in (14). The degrees $D_{g}$ and $D_{h}$ of the penalty polynomials depend on the desired precision of the time delay estimate that determines the passband ripple and transition bandwidth of $\Pi(z)$. Typically, they are much smaller than $D_{f}$. The choice of penalty function is analogous to the choice of penalty in the penalized maximum likelihood algorithm.

\section{Iterative Penalized Maximum Likelihood (PML)}

The penalized ML estimate of a $\theta=\left[a, d, b_{0}, \cdots, b_{N-1}\right]^{T}$ is defined as

$$
\hat{\theta}=\arg \max _{\theta \in \Theta}\{\log p(Y ; \theta)-P(\theta)\} .
$$

Here, $P(\theta)$ is a user-specified penalty function. Penalties have frequently been introduced to regularize the estimator [40], to promote faster convergence [14], or to take advantage of prior information [39]. It will be convenient to express the penalty function as the function $P(z)=-\log \Pi(z)$ of the complex variable $z=e^{j \omega_{0} d}$ defined in (13). In this way, it can be seen that incorporation of the aforementioned polynomial rooting estimation procedure for estimation of $d$ is equivalent to implementing a PML solution to the joint estimation of $\theta$.

In cases where direct maximization of the objective (17) is not possible, iterative optimization is often useful. The iterative algorithm developed in this paper is a coordinate ascent-type algorithm that is related to Gauss-Seidel iterations [18] for minimizing quadratic objectives. Fix a coordinate $\theta_{i}$ to be updated, and define the parameter vector $\theta_{-i}$ as $\theta$ with the coordinate $\theta_{i}$ left out: $\theta_{-i}=\left[\theta_{1}, \cdots, \theta_{i-1}, \theta_{i+1}, \cdots\right.$, $\left.\theta_{N+2}\right]^{T}$. Define $j_{i}$ as a mapping from the natural numbers $1,2, \cdots$ to the parameter index set $\{1, \cdots, N+2\}$ that satisfies the property that the sequence $j_{i}, i=1,2, \cdots$ cycles through all parameter indices an infinite number of times. For $i=1,2, \cdots$, the coordinate ascent algorithm produces a sequence $\left\{\theta^{(i)}\right\}_{i=1}^{\infty}$ by the following iteration:

$$
\begin{aligned}
\theta_{j_{i}}^{(i)} & =\arg \max _{\theta_{j_{i}}}\left\{\log p\left(Y ; \theta_{j_{i}}, \theta_{-j_{i}}^{(i-1)}\right)-P\left(\theta_{j_{i}}, \theta_{-j_{i}}^{(i-1)}\right)\right\} \\
\theta_{-j_{i}}^{(i)} & =\theta_{-j_{i}}^{(i-1)} .
\end{aligned}
$$

Note that in the $i$ th iteration, only the parameter $\theta_{j_{i}}$ of $\theta$ is updated while the other parameters $\theta_{-j_{i}}$ are held fixed.

For the present application, the penalized log likelihood function is of the form $\log p(Y ; \theta)+\log \Pi\left(e^{j \omega_{0} d}\right)$, where $\log p(Y ; \theta)$ is given by (5). The convergence rate of coordinate ascent depends on the order of parameter updates and the number of consecutive updates of a given parameter. For positive integers $k, N$, denote by $[k]_{N}$ the integer $k$ modulo
$N$. Given initial conditions $a^{(0)}, d^{(0)}, b_{0}^{(0)}, \cdots, b_{N-1}^{(0)}$, the following is an algorithm that updates in the order $d^{(i)} \rightarrow$ $d^{(i+1)}, b_{[i-1]_{N}}^{(i)} \rightarrow b_{[i-1]_{N}}^{(i+1)} a^{(i)} \rightarrow a^{(i+1)}$. Here, the notation $[i-1]_{N}$ denotes $(i-1) \bmod N$.

1) Increasing Complexity Single-User Algorithm: For $i=$ $0,1, \cdots$, update $i$ th cycle.

1) Find $d^{(i+1)}$ by rooting the polynomial $\phi_{a^{(i)}, \underline{b}^{(i)}}(z)$ as explained in previous section.

2) Update the bit $b_{[i-1]_{N+2}}$ by hard decision

$$
\begin{aligned}
& b_{[i-1]_{N}}^{(i+1)}= \operatorname{sign}\left(\operatorname { R e } \left\{a^{(i)} \sum_{k} w_{k}\left([i-1]_{N+2} ; d^{(i)}\right)\right.\right. \\
&\left.\left.\cdot\left[Y_{k}^{*}-a(i) * I_{k}^{(i) *}\left([i-1]_{N+2}\right)\right]\right\}\right) .
\end{aligned}
$$

where

$$
I_{k}^{(i)}(n)=\sum_{n^{\prime}=0, n^{\prime} \neq n}^{N-1} b_{n^{\prime}}^{(i)} w_{k}\left(n^{\prime} ; d^{(i)}\right) .
$$

3) Update the amplitude $a$ as follows. Define temporary variable

$$
\hat{a}=\frac{\sum_{k} Y_{k} \sum_{n=0}^{N-1} b_{n}^{(i) *} w_{k}^{*}\left(n ; d^{(i)}\right)}{\sum_{k}\left|\sum_{n=0}^{N-1} b_{n}^{(i)} w_{k}\left(n ; d^{(i)}\right)\right|^{2}} .
$$

If $0 \leq \arg (\hat{a})<\pi$, set $a^{(i+1)}=\hat{a}$ and $b_{n}^{(i+1)}=b_{n}^{(i)}$ for all $n \in I_{N}$; otherwise, set $a^{(i+1)}=-\hat{a}$, and $b_{n}^{(i+1)}=-b_{n}^{(i)}$, for all $n \in I_{N}$.

Note that since, at each iteration, the objective function is maximized over the associated coordinate, the algorithm ensures a monotone increase in likelihood as it progresses. Use of the temporary variable $\hat{a}$ is necessary to ensure that $a$ has non-negative phase. A deficiency of the algorithm is the problem of growing memory and computation. It must cycle over an increasingly large set of symbols as the number $N$ of these increases.

\section{COMPLEXITy REDUCTION VIA WAVELET BASIS}

The growing memory and growing complexity problem will be solved by prescribing a single-pass acyclic version of the PML algorithm that updates only those symbols falling within a sliding time window of fixed length. In this scheme, the time localized parameters, i.e., symbols $b_{n}$, are only updated a finite number of times, whereas the global parameters, i.e., $a$ and $d$, are updated an infinite number of times. Recall that in Section II, a general orthogonal representation for the received waveform $\{Y(t)\}_{t \in[0, T]}$ was used, whereby projecting it on an orthogonal basis $\left\{\psi_{k}(t)\right\}_{k>0}$, an equivalent set of measurements $Y_{k}=\left\langle Y(\cdot), \psi_{k}(\cdot)\right\rangle, k=1,2, \cdots$ was obtained. To achieve decoupling between the localized and nonlocalized parameter updates, it will be convenient to specialize the basis $\left\{\psi_{k}\right\}$ to one that has the multiresolution property [25]. This 


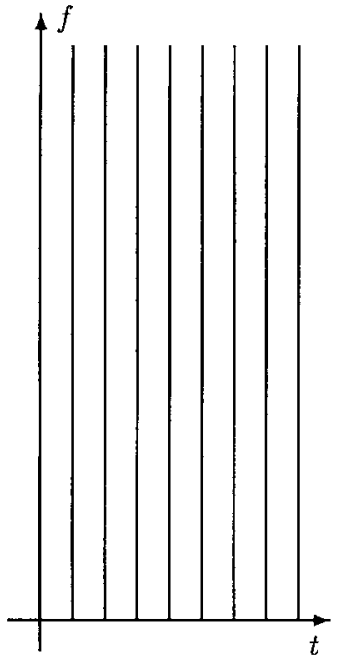

(a)

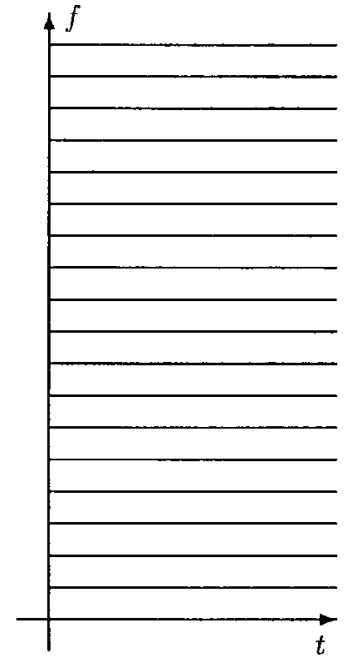

(b)

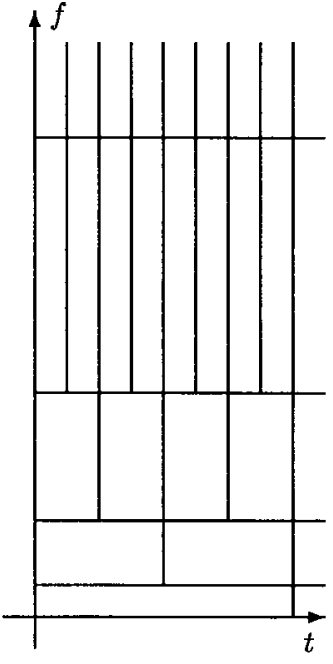

(c)

Fig. 1. Tiling of the time frequency plane. (a) Dirac basis, (b) Fourier basis. (c) Wavelet basis.

will produce coefficients $Y_{k}$ that contain only information specific to a particular time and scale component of $\{Y(t)\}_{t \in[0, T]}$. In this way, a kind of parsimony of the data representation is achieved. Information needed for a particular local or nonlocal parameter update is concentrated in only a few coefficients $Y_{k}$.

Fig. 1 shows time scale tiling diagrams of three orthonormal bases: the Dirac basis, the Fourier basis, and the wavelet basis. While the Dirac and Fourier bases are localized only in the time and frequency dimensions, respectively, the wavelet basis has the multiresolution property. Each basis is localized in both dimensions, with a timing resolution that gets finer at smaller scales. The time-localized Dirac basis is not parsimonious for updating parameters that are localized in frequency. On the other hand, the Fourier basis is not parsimonious for updating time localized parameters such as the symbol sequence. The same deficiencies would hold for other bases that do not have the multiresolution property, e.g., the cardinal series basis, the Slepian (prolate spheroid) basis, and the Walsh basis.

Note that there exist nonorthonormal expansions that also have the time-frequency resolution property. For example, Gabor frames [7] offer more regular tiling of the time-frequency plane. However, nonorthogonality causes leakage across scales and complicates the maximization of the likelihood function. Among the many wavelet bases that can be used, e.g., the Daubechies wavelets, Battle-Lemarie wavelets, wavelet packets [8], [42], local cosine bases, and biorthogonal wavelets [7], in this paper, the Daubechies wavelet basis [6] is adopted. An advantage of this basis is that the coefficients can be computed in real time using the discrete time wavelet transform (DTWT) algorithm [35].

The discrete wavelet basis is defined by time scaling and translation of the single function, which is called the basic wavelet $\psi(t)$ [8], producing the double indexed set of basis elements

$$
\psi_{j k}(t)=2^{-j / 2} \psi\left(2^{-j} t-k\right) \quad(j, k) \in \mathbb{Z}^{2} .
$$

Use of the wavelet basis produces a double indexed set of equivalent measurements $Y_{j k}=\left\langle Y(\cdot), \psi_{j k}(\cdot)\right\rangle,(j, k) \in \mathbb{Z}^{2}$, signaling waveform projections $w_{j k}(n ; d)=\left\langle p\left(\cdot-n T_{b}-d\right)\right.$, $\left.\psi_{j k}(\cdot)\right\rangle$, and a triple indexed set of Fourier coefficients $c_{j k, m}$ of the projections, as defined in the previous section.

As only certain subsets of the wavelet coefficients will be used for each parameter update, it is convenient to define an increasing sequence of subsets of wavelet indices $\left\{W^{(i)}\right\}_{i=0}^{\infty}$ such that

$$
\begin{aligned}
& W^{(i)} \subset W^{(i+1)} \quad i \geq 0 \\
& \bigcup_{i} W^{(i)}=\mathbb{Z}^{2} .
\end{aligned}
$$

The following conditions will be necessary

i) The signaling waveform $p(t)$ and the basic wavelet $\psi(t)$ are supported on $\left[-T_{p} / 2, T_{p} / 2\right]$ and $\left[-T_{\psi} / 2, T_{\psi} / 2\right]$, respectively, for some $T_{p}$ and $T_{\psi}$, where $T_{p} \geq T_{b}$.

ii) A finite number of scales is used in the wavelet decomposition so that the scale index $j$ satisfies $q^{\prime} \leq j \leq q$ for some $q^{\prime}, q \in \mathbb{Z}$.

iii) The wavelet index sets $W^{(i)}$ used in the algorithm are chosen sequentially in such a way that

$$
\begin{aligned}
& \min \left\{2^{j} k:(j, k) \in W^{(i+1)}-W^{(i)}\right\} \\
& \geq \max \left\{2^{j} k:(j, k) \in W^{(i)}\right\}
\end{aligned}
$$

for all $i \in \mathbb{N}$.

The purpose of these assumptions, which are not restrictive, will now be explained. The first assumption is needed to eliminate all but a small neighborhood of overlapping symbols $\left\{b_{n}\right\}$ appearing in the likelihood function. The second assumption is required so that the Fourier coefficients $c_{j k, m}(n)$ can be stored in a table of finite size and that the algorithm have a finite delay. The smallest scale $q^{\prime}$ can be determined from the bandwidth of the signaling waveform $p(t)$, and the largest scale $q$ can be determined by the maximum tolerable processing delay, e.g., $T$. The third condition means that the wavelet coefficients are processed over all scales simultaneously and sequentially in time according to increasing temporal localization variable 


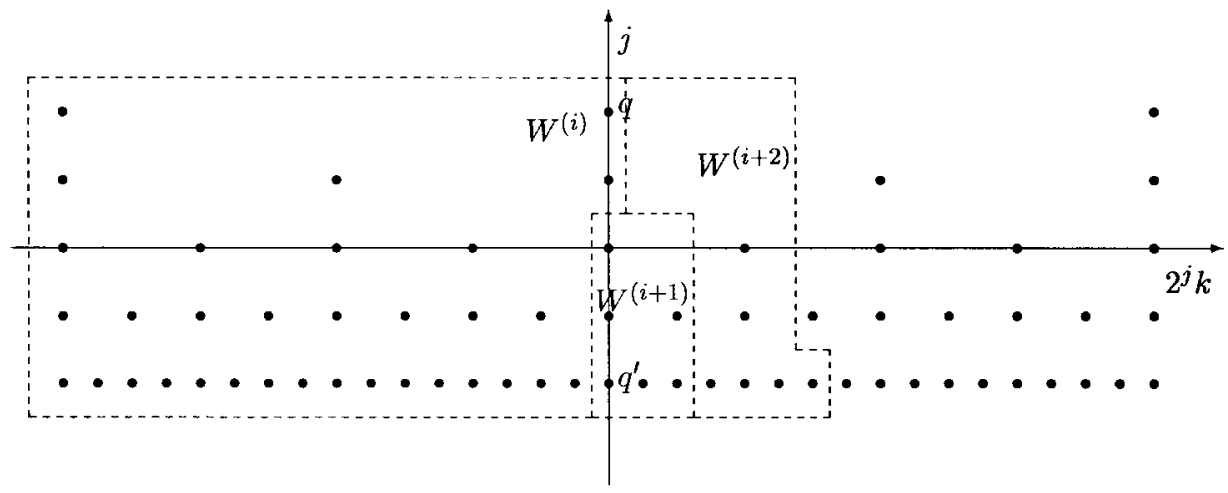

Fig. 2. Wavelet index sets.

$2^{j} k$. A graphical illustration of the actual sequence of wavelet indices used in the algorithm is given in Fig. 2.

It is useful to remark that if $T_{b}=r 2^{q}$ for some rational number $r=r_{1} / r_{0}$, then it is possible to store all the Fourier coefficients $c_{j k, m}(n)$ in a finite table because they can all be mapped to those with symbol indices $0, \cdots, r_{0}-1$

$$
c_{j k, m}(n)=c_{j k-n_{0} r_{1} 2^{q-j}, m}\left(n_{1}\right), \quad 0 \leq n_{1}<r_{0}
$$

where $n_{1}=n\left(\bmod r_{0}\right)$.

Next, define the following intermediate variables, which will be updated at each iteration of the algorithm:

$$
\begin{aligned}
\mu_{m}^{(i)}(n) & =\sum_{j k \in W^{(i)}} Y_{j k} c_{j k, m}^{*}(n) \quad m \in \mathbb{Z}, n \in I_{N} \\
\nu_{m}^{(i)}\left(n_{1}, n_{2}\right) & =\sum_{j k \in W^{(i)}} d_{j k, m}\left(n_{1}, n_{2}\right) \\
m \in \mathbb{Z}, n_{1}, n_{2} \in I_{N} &
\end{aligned}
$$

where

$$
d_{j k, m}\left(n_{1}, n_{2}\right)=\sum_{p \in \mathbb{Z}} c_{j k, p}\left(n_{1}\right) c_{j k, p-m}^{*}\left(n_{2}\right) .
$$

Similar to the Fourier coefficients $c_{j k, m}(n)$, the constants $d_{j k, m}\left(n_{1}, n_{2}\right)$ can be stored in a finite table. It is easy to verify that $\nu_{m}^{(i)}\left(n_{1}, n_{2}\right)$ satisfies the symmetry properties

$$
\begin{gathered}
\nu_{m}^{(i)}\left(n_{1}, n_{2}\right)=\nu_{m}^{(i)}\left(n_{2}, n_{1}\right) \\
\nu_{-m}^{(i)}\left(n_{1}, n_{2}\right)=\nu_{m}^{(i) *}\left(n_{1}, n_{2}\right) .
\end{gathered}
$$

Notice that $\mu_{m}^{(i)}(n)$ is the resultant matched filtering of $\left\{Y_{j k}\right\}$ to the Fourier coefficients of the correlation $w_{j k}(n ; d)$, whereas $\nu_{m}^{(i)}\left(n_{1}, n_{2}\right)$ is a deterministic quantity.

To reduce the computational complexity of updating the intermediate variables $\mu$ and $\nu$, it is useful to exploit the finite support property of the basic wavelet. To this aim, define the quantity

$$
B=\left\lceil\left(\frac{T_{p}+T_{0}}{2}+2^{q-1} T_{\psi}\right) / T_{b}\right\rceil
$$

where $\lceil x\rceil$ indicates the smallest integer that is greater than or equal to $x$. Recall that $T_{p}, T_{\psi}$, and $T_{0}$ are the signaling pulse width, the width of the basic wavelet, and the Fourier analysis window length defining the coefficients $c_{j k, m}$, respectively.
In addition, for a wavelet index set $W^{(i)}$, define the indices $n_{-}^{(i)}$ and $n_{+}^{(i)}$

$n_{-}^{(i)}=\min \left\{n: n \geq \frac{2^{j} k}{T_{b}}\right.$, for all $\left.(j, k) \in W^{(i)}, n \in \mathbb{Z}\right\}$

$n_{+}^{(i)}=\max \left\{n: n \leq \frac{2^{j} k}{T_{b}}\right.$, for some $\left.(j, k) \in W^{(i)}, m \in \mathbb{Z}\right\}$.

Proposition 1 of the Appendix shows that $B$ is a timewidth parameter for $\nu_{m}$ in the sense that $\nu_{m}^{(i)}\left(n_{1}, n_{2}\right)=0$ for all $m$ for $\left|n_{1}-n_{2}\right| \geq 2 B$. Proposition 2 of the Appendix shows that $\nu_{m}^{(i)}\left(n_{1}, n_{2}\right)=0$ whenever $n_{1}$ or $n_{2}$ exceed $n_{-}^{(i)}+B$ and $\mu_{m}^{(i)}(n)=0$ whenever $n$ exceeds $n_{-}^{(i)}+B$ for all $m \in \mathbb{Z}$. The combination of these two propositions specifies a small region of wavelet indices for which the summands of $\mu_{m}^{(i)}$ and $\nu_{m}^{(i)}$ are nonzero. Proposition 3 of the Appendix shows that if $n_{1} \leq n_{+}^{(i)}-B$ or $n_{2} \leq n_{+}^{(i)}-B$, then $\nu_{m}^{(p)}\left(n_{1}, n_{2}\right)=\nu_{m}^{(i)}\left(n_{1}, n_{2}\right)$ for all $p \geq i$ and all $m \in \mathbb{Z}$ and similarly for $\mu_{m}^{(i)}$. Thus, this specifies a set of indices for which these intermediate variables need not be updated.

Once the following update index sets are defined, a concrete specification of the algorithm can be given.

$$
\begin{aligned}
& T^{(i)}=\left\{n: n_{+}^{(i-1)}-B<n<n_{-}^{(i)}+B\right\} \\
& U^{(i)}=\left\{n: n_{+}^{(i-1)}-B<n \leq n_{+}^{(i)}-B\right\} .
\end{aligned}
$$

Notice that the set $T^{(i)}$ is not empty because $n_{-}^{(i)} \geq n_{+}^{(i-1)}$ and $B \geq 1$, whereas the set $U^{(i)}$ may be empty. Corresponding to the above sets, define the two-dimensional (2-D) index sets

$$
\begin{aligned}
R^{(i)}= & \left\{\left(n_{1}, n_{2}\right): n_{1} \in T^{(i)}, n_{1}-2 B<n_{2} \leq n_{1}\right\} \\
& \cup\left\{\left(n_{1}, n_{2}\right): n_{2} \in T^{(i)}, n_{2}-2 B<n_{1} \leq n_{2}\right\} \\
V^{(i)}= & \left\{\left(n_{1}, n_{2}\right): n_{1} \in U^{(i)}, n_{1}-2 B<n_{2} \leq n_{1}\right\} \\
& \cup\left\{\left(n_{1}, n_{2}\right): n_{2} \in U^{(i)}, n_{2}-2 B<n_{1} \leq n_{2}\right\} .
\end{aligned}
$$

With the above, a fourth condition can be specified on the sliding symbol-update window.

iv) During the $i$ th iteration, the algorithm only updates the symbols $\left\{b_{n}: n \in T^{(i)}\right\}$. 
Note that for symbols not in $T^{(i)}$, the intermediate variables do not change as a result of the most recent multiresolution samples having indices in $W^{(i)}-W^{(i-1)}$. Hence, the only change in the objective of these symbols is a result of updating other parameters, which should have a secondary effect. Consequently, the algorithm no longer will have the cyclic update structure that it had before in Section III. The set $T^{(i)}$ can therefore be referred to as the "current symbols" index set, whereas the set $U^{(i)}$ corresponds to "past symbols," that is, symbols whose estimates will not be further updated. The 2-D sets $R^{(i)}$ and $V^{(i)}$ that define pairs of symbol indices can be interpreted in a similar manner.

The fixed memory wavelet version of the coordinate ascent algorithm presented in Section III is given below. To simplify the notation, the Fourier index will be omitted, and the corresponding variables will be typed in boldface, e.g., $\boldsymbol{c}_{j k}(n)$ instead of $c_{j k, m}(n)$.

1) Fixed Complexity Single User Algorithm: For $i=$ $0,1, \cdots$

1) Choose the wavelet index set $W^{(i+1)}$.

2) Update the local variables $\boldsymbol{\mu}^{(i)}$ and $\boldsymbol{\nu}^{(i)}$ as

$$
\begin{aligned}
& \boldsymbol{\mu}^{(i+1)}(n)=\boldsymbol{\mu}^{(i)}(n)+\sum_{j k \in D^{(i+1)}} y_{j k} \boldsymbol{c}_{j k}^{*}(n) \\
& \boldsymbol{\nu}^{(i+1)}\left(n_{1}, n_{2}\right)=\boldsymbol{\nu}^{(i)}\left(n_{1}, n_{2}\right)+\sum_{j k \in D^{(i+1)}} \boldsymbol{d}_{j k}\left(n_{1}, n_{2}\right) \\
& n_{1}, n_{2} \in T^{(i+1)},\left|n_{1}-n_{2}\right|<2 B
\end{aligned}
$$

where $D^{(i+1)}=W^{(i+1)}-W^{(i)}$.

3) Update the state variables $\boldsymbol{\alpha}^{(i)}$ and $\boldsymbol{\beta}^{(i)}$ as

$$
\begin{aligned}
& \boldsymbol{\alpha}^{(i+1)}=\boldsymbol{\alpha}^{(i)}+\sum_{\left(n_{1}, n_{2}\right) \in V^{(i)}} b_{n_{1}}^{(i)} b_{n_{2}}^{(i) *} \boldsymbol{\nu}^{(i)}\left(n_{1}, n_{2}\right) \\
& \boldsymbol{\beta}^{(i+1)}=\boldsymbol{\beta}^{(i)}+\sum_{n \in U^{(i)}} b_{n}^{(i) *} \boldsymbol{\mu}^{(i)}(n) .
\end{aligned}
$$

4) Update the delay estimate $d$.

- Compute the temporary variables

$$
\begin{aligned}
& \gamma^{(i+1)}=\left|a^{(i)}\right|^{2}\left[\boldsymbol{\alpha}^{(i+1)}+\sum_{\left(n_{1}, n_{2}\right) \in R^{(i+1)}}\right. \\
& \left.\cdot b_{n_{1}}^{(i)} b_{n_{2}}^{(i) *} \nu^{(i+1)}\left(n_{1}, n_{2}\right)\right] \\
& \delta^{(i+1)}=a^{(i) *}\left[\beta^{(i+1)}+\sum_{n \in T^{(i+1)}} b_{n}^{(i) *} \mu^{(i+1)}(n)\right] .
\end{aligned}
$$

Set up the penalized objective for $z=e^{i \omega_{0} d}$.

$$
\begin{aligned}
\phi^{(i+1)}(z)= & \frac{2}{N_{0}} \sum_{|m| \leq D_{f}}\left(\delta_{-m}^{(i+1)}+\delta_{m}^{(i+1) *}-\gamma_{m}^{(i+1)}\right) z^{m} \\
& +\log \Pi(z) .
\end{aligned}
$$

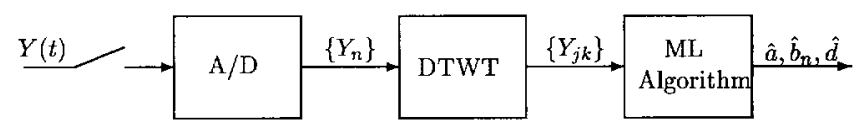

Fig. 3. Block diagram of the ML receiver.

- Maximize the penalized objective using polynomial rooting

$$
z^{(i+1)}=\underset{z=e^{i \omega}}{\operatorname{argmax}}\left\{\phi^{(i+1)}(z)\right\} .
$$

5) Update symbol estimates $b_{n}, n \in T^{(i+1)}$.

- Update the local variable

$$
\epsilon^{(i+1)}(n)=\epsilon^{(i)}(n)+\sum_{\substack{n_{2} \in U^{(i)} \\\left|n-n_{2}\right|<2 B}} b_{n_{2}}^{(i) *} \nu^{(i)}\left(n, n_{2}\right) .
$$

Next, include the most recent data samples

$$
\begin{aligned}
\varepsilon^{(i+1)}(n)= & a^{(i)} \sum_{|m| \leq D_{f}}\left[\mu_{m}^{(i+1) *}(n)-a^{(i) *}\left(\epsilon_{m}^{(i+1)}(n)\right.\right. \\
& \left.+\sum_{\substack{n_{2} \in T^{(i+1)} \\
n_{2} \neq n}} b_{n_{2}}^{(i) *} \nu_{m}^{(i+1)}\left(n, n_{2}\right)\right] z^{(i) m} .
\end{aligned}
$$

The objective for $\left\{b_{n}\right\}$ is (need not compute)

$$
Q^{(i+1)}\left(b_{n}\right)=\frac{2}{N_{0}}\left[b_{n} \varepsilon^{(i+1)}(n)+b_{n}^{*} \varepsilon^{(i+1) *}(n)\right] .
$$

- Maximize (hard decision)

$$
b_{n}^{(i+1)}=\operatorname{sign}\left[\operatorname{Re}\left(\varepsilon^{(i+1)}(n)\right)\right] .
$$

6) Update the amplitude estimate $a$.

- Compute the temporary variables

$$
\begin{aligned}
& \zeta^{(i+1)}=\sum_{|m| \leq D_{f}} z^{(i) m} \\
& \cdot\left(\beta_{-m}^{(i+1)}+\sum_{n \in T^{(i+1)}} b_{n}^{(i) *} \mu_{-m}^{(i+1)}(n)\right) \\
& \eta^{(i+1)}=\sum_{|m| \leq D_{f}}\left(\alpha_{m}^{(i+1)}+\sum_{\left(n_{1}, n_{2}\right) \in R^{(i+1)}}\right. \\
& \left.\cdot b_{n_{1}}^{(i)} b_{n_{2}}^{(i) *} \nu_{m}^{(i+1)}\left(n_{1}, n_{2}\right)\right) z^{(i) m} \text {. }
\end{aligned}
$$

The objective for $a$ is (need not compute)

$$
Q^{(i+1)}(a)=a^{*} \zeta^{(i+1)}+a \zeta^{(i+1) *}-|a|^{2} \eta^{(i+1)} .
$$

- Maximize the objective. Let

$$
\hat{a}=\zeta^{(i+1)} / \eta^{(i+1)} .
$$




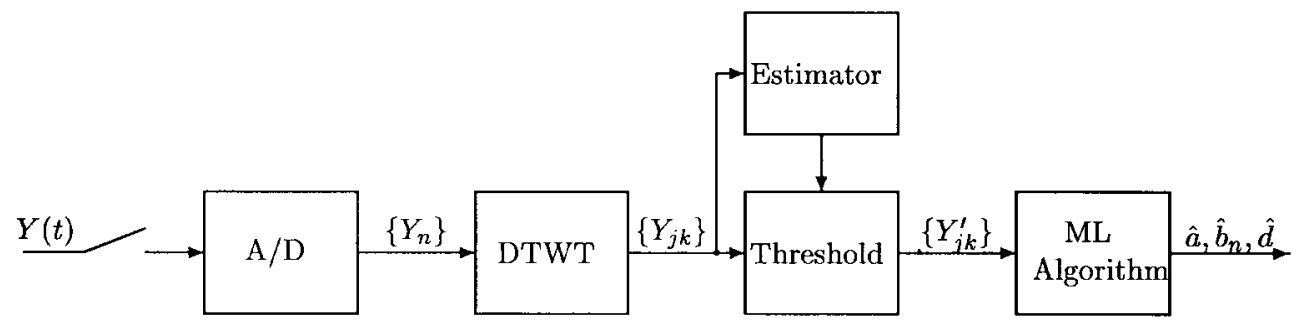

Fig. 4. Block diagram of the wavelet thresholding receiver.

If $0 \leq \arg (\hat{a})<\pi$, set $a^{(i+1)}=\hat{a}$ and $b_{n}^{(i+1)}=b_{n}^{(i)}$ for all $n \in I_{N}$; otherwise, set $a^{(i+1)}=-\hat{a}, b_{n}^{(i+1)}=-b_{n}^{(i)}$ for all $n \in I_{N}$, and multiply $\beta^{(i+1)},\left\{\epsilon^{(i+1)}(n): n \in T^{(i+1)}\right\}$ by -1 .

Notice that inversion of the signs of all the symbols, as implied by the last step, can be most easily done by keeping track of the inversions and performing it just once after the final iteration. In this way, multiple writes to memory are avoided.

An implementation of the PML receiver is shown by the block diagram in Fig. 3. The incoming complex baseband signal $y(t)$ is oversampled and digitized. The oversampling should be sufficiently high so that the discrete wavelet transform in continuous time is well approximated by a discrete time wavelet transform. The sampled sequence $\left\{y_{n}\right\}$ is wavelet transformed using the DTWT algorithm [35], which can be efficiently implemented with an octave-band filter bank structure. The fully digital algorithm described above performs ML estimation of the parameters in a time recursive manner and passes the estimated symbol sequence $\hat{b}_{n}$ on to further decoding stages.

\section{IMPULSIVE NOISE ROBUSTIFICATION}

In this section, a robustification of the algorithm is given for impulsive noise channels. The wavelet shrinkage method of [10] uses a soft wavelet shrinkage algorithm to optimally reconstruct a signal from samples contaminated by additive white Gaussian noise. In this method, small wavelet coefficients are set to zero since they are likely to contain little signal energy, and, to compensate, larger wavelet coefficients are scaled down since they are likely to contain greater signal energy. This method, similarly to [1], eliminates noisy wavelet coefficients by comparison to a predetermined high threshold. Wavelet coefficients larger than the threshold are rejected because they have most likely been corrupted by the impulsive events. In the method of [1], the rejected wavelet coefficients are reconstructed via an FFT-based interpolation algorithm. Assuming that the noise is dominated by the impulsive component while the Gaussian noise is very weak, there is practically no loss of performance if the noisy coefficients are rejected along with the signal component. This is because the residual signal power is still very large compared with the Gaussian noise power. While such an extension is not pursued in this paper, we could also simultaneously implement soft thresholding to reduce the effect of high power Gaussian noise.

A general block diagram of the wavelet-based impulsive noise receiver is shown in Fig. 4. This receiver is very similar to the optimum receiver for the AWGN channel described in
Section IV. The additional blocks in the diagram perform the threshold estimation algorithm proposed in [1] and the wavelet thresholding operation. In the threshold estimation algorithm, the coefficient sequence in each wavelet band is divided into segments, whose length is determined by the length of the time interval where the signal is assumed locally stationary. In each signal segment, the standard deviation of the signal is estimated by the square root of the sample variance. The threshold at scale index $j, T h_{j}$ is set to a fixed multiple of the standard deviation estimate in that band. At the finer scales, the impulsive events are isolated, i.e., no two impulses are captured by a single wavelet coefficient. In the coarser scales, the impulses are no longer isolated; therefore, the threshold should be decreased in order to effectively detect the corrupted coefficients. This can be done by multiplying the threshold with a slowly decreasing function of scale. The wavelet thresholding operator generates a new wavelet coefficient sequence $\left\{Y_{j k}^{\prime}\right\}$ as follows:

$$
Y_{j k}^{\prime}= \begin{cases}Y_{j k} & \left|Y_{j k}\right|<T h_{j} \\ 0 & \left|Y_{j k}\right| \geq T h_{j} .\end{cases}
$$

The rest of the algorithm remains as before.

\section{SimULATIONS}

The algorithm of Section IV has been evaluated by means of a simulation program written in MATLAB. Several system parameters can be varied in order to examine their effect on the receiver's performance, e.g., choice of wavelet family, number of scales, step size, extent of ISI, choice of PN codes, and number of data symbols. The performance criteria of interest were the symbol error probability and root mean squared (RMS) error of the time delay and phase estimates.

In this paper, only a small subset of all possible variations of system parameters will be presented. We have restricted our attention to an uncoded single-user system at the low SNR range of -1 to $8 \mathrm{~dB}$. The bit error probability results were obtained from simulation runs on contiguous data blocks of 2048 or 4096 bits each. The gain and time delay estimates converged within the first 90 bits of each data block. Afterwards, no further updates of these parameters were necessary. The phase and synchronization error performance results were obtained from 100 Monte Carlo simulations on 32 bit long data blocks. The signal parameters were chosen randomly and independently of each other. The data bits $\left\{b_{n}\right\}$ were selected as either +1 or -1 , and the time delay $d$ was uniformly distributed in $\left[-T_{b} / 2, T_{b} / 2\right]$. 
A seven-chip PN code was used for the spreading sequence. The transmitted signal was passed through a band-limited AWGN channel $|f| \leq 1 / 2 T_{c}$, where $T_{c}$ is the chip time. The Daubechies wavelets [6] of length 4, which are the shortest continuous compactly supported wavelets, were used in the wavelet decomposition of the received signal. The decomposition was done on six scales in order to capture a large percentage of the signal energy, close to $90 \%$ on the average. The number of new wavelet coefficients added per cycle, i.e., the size of $W^{(i+1)}-W^{(i)}$, was 11 , and the number of wavelet samples per bit interval was approximately 63 . The time-delay penalty $\Pi(z)$ was designed using a sixth-order Chebychev equiripple filter. The simulations focus on aided acquisition or tracking performance as opposed to general acquisition performance to reduce the additional complications of local maxima in the likelihood function. Hence, the time delay estimate was initialized close to the true parameter by adjusting the delay penalty function so that the maximum with respect to $d$ is sought within $70 \%$ of $T_{c}$ from the true time delay. Similar procedure was adopted to ensure proper phase initialization during the first four symbols of each transmission. The issue of global phase and synchronization acquisition should be addressed in a future study.

Fig. 5 shows the bit error probability for the AWGN channel. The results were obtained from approximately 8000 simulated bits at the highest SNR value to 2000 bits at the lowest SNR. We observe that the simulation results closely match the theoretical lower bound on probability of error for a BPSK decoder [24]. The performance of a phase-coherent DLL with comparable response time is also shown. The parameter $\delta=2 / W_{L} T$ is the normalized response time of the DLL, where $W_{L}$ is the two-sided loop bandwidth, and $T$ is the signaling period; therefore, a value of $\delta=31$ was used. The loop damping of the equivalent PLL was taken as $\zeta=0.707$, and zero detuning was assumed. The DLL error probability was found by numerical integration of the conditional PSK bit error probability with respect to the solution of a stochastic PDE of the steady-state synchronization error [24]. Notice that the DLL performance has degraded by more than $1 \mathrm{~dB}$ with respect to the ideal PSK error bound, whereas the performance of the coordinate ascent algorithm is essentially optimal. Fig. 6 shows the normalized synchronization performance of the coordinate ascent algorithm $\left(T_{b}=1\right)$. The coordinate ascent algorithm achieves an RMS synchronization error that is much smaller than the chip time of $T_{c} \approx 0.14$, which explains its nearly optimal symbol estimation performance. The synchronization error of the DLL is seen to be much larger. The Cramér-Rao (CR) bound on time delay estimation is also shown for reference. Fig. 7 compares the RMS phase error of the coordinate ascent algorithm with that of a data aided loop (DAL). The CR bound on phase error is shown for reference [36]. The DAL performance was calculated with the same value of $\delta$ as above for the case of a suppressed carrier with NRZ data ( $m=0$, see [23]). The two systems have a similar performance, but it should be noted that the DAL relies on a perfectly synchronized reference.

Finally, impulse noise channel results are shown in Fig. 8. The impulses arrived according to a Poisson process with an

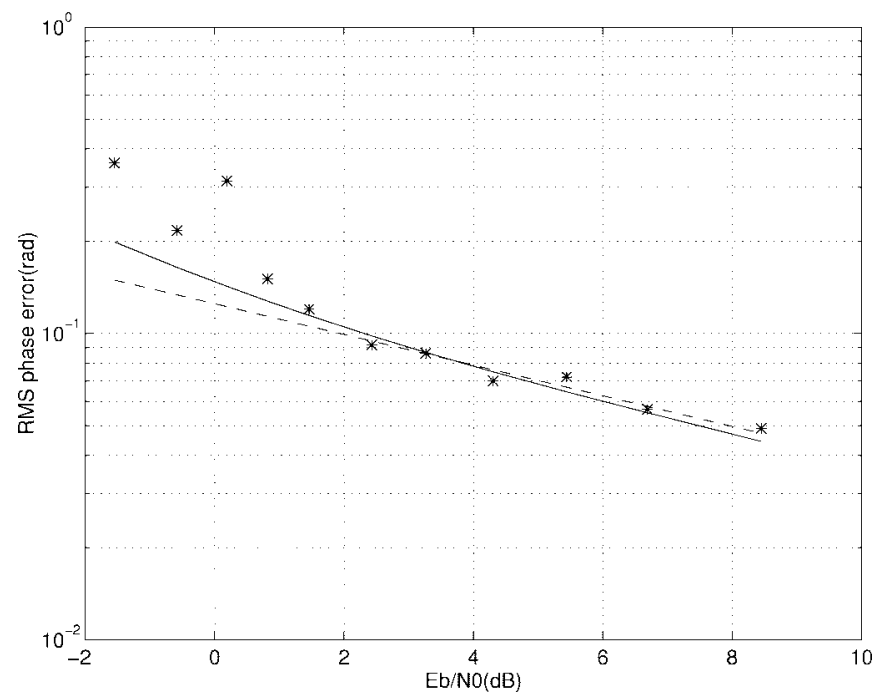

Fig. 5. Bit error probability versus SNR for Gaussian noise channel. Stars: simulation results. Solid: DLL performance, Dotted: PSK error bound.

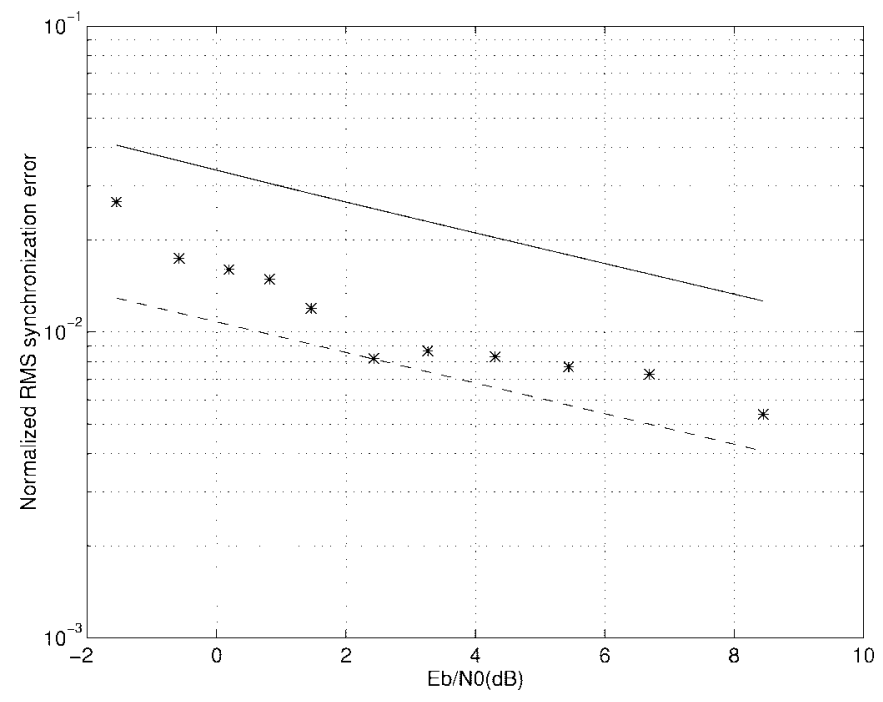

Fig. 6. Normalized RMS synchronization error versus SNR. Stars: simulation result. Solid: DLL performance. Dashed: CR bound.

average inter-arrival time of approximately $1.5 T_{b}$. The impulse amplitudes were generated according to an i.i.d. complex Gaussian process, and the width of each impulsive event was equal to the chip time $T_{c}$, thus effectively covering the signal spectrum. In addition, a weak background AWGN process whose power was $18 \mathrm{~dB}$ below signal power was present. The thresholds were computed by the following simple iterative algorithm. First, the standard deviation $\sigma$ of each scale is estimated, and then, coefficients whose magnitude is larger than $3 \sigma$ are removed, and the process is repeated using the remaining coefficients until convergence. The thresholds were scaled by dividing by $\sqrt{j}$, where the scale index $j$ ranged between 1 to 6 . The simulations compared bit error probability estimates of the conventional receiver with those of the wavelet thresholding receiver over a range of signal-to-impulse ratio (SIR). The figure shows that a marked improvement in bit error probability has been achieved by using the wavelet thresholding technique coupled with the coordinate ascent algorithm. 


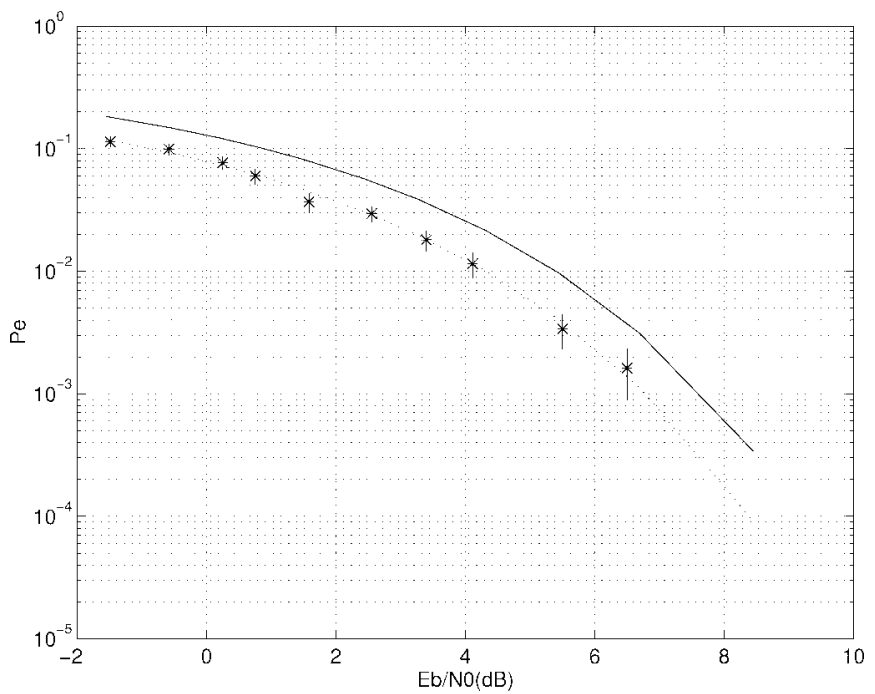

Fig. 7. RMS phase error. Stars: simulation results. Solid: DAL performance. Dashed: CR bound.

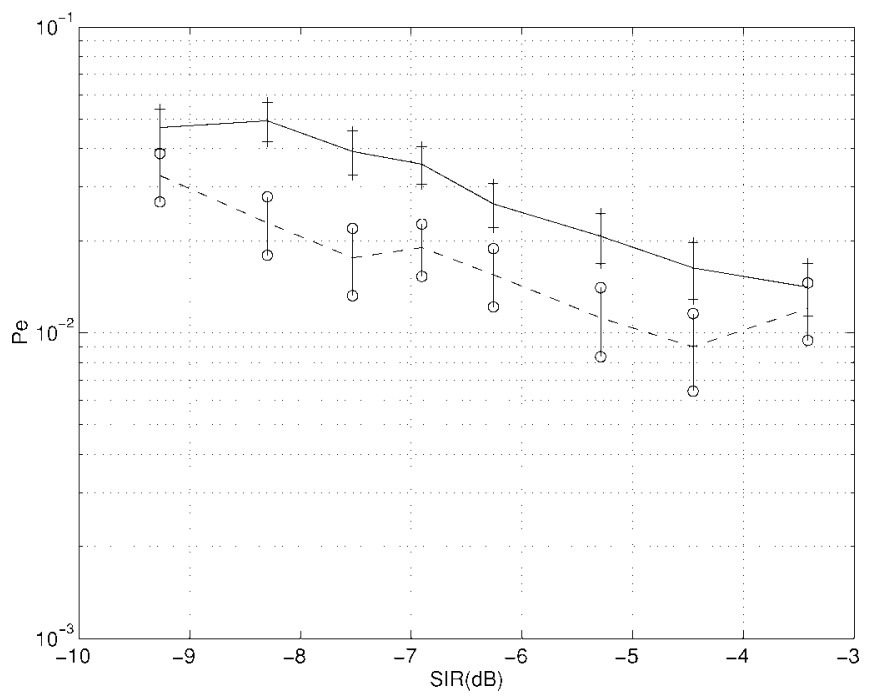

Fig. 8. Bit error probability versus SIR for impulsive noise channel. Solid. Conventional receiver. Dashed: Wavelet thresholding receiver. Confidence intervals are marked with "+" and "o," respectively.

\section{CONCLUSIONS}

This paper introduces a new grouped coordinate ascent method for joint timing and phase synchronization and optimal ML detection of transmitted symbols in a single user receiver. Wavelet thresholding can also be incorporated to adapt the receiver to impulsive noise interference channels. Fourier series and polynomial rooting were used to simplify the delay parameter line search, and a multiresolution wavelet representation of the received signal was used to efficiently match parameter updates to data coefficients. The flexibility in choosing several system parameters such as the wavelet basis, the step size of the algorithm, and the penalty functions, makes the algorithm suitable for a variety of applications, depending on technological feasibility and cost considerations. It should be pointed out that the problem of selecting an optimal wavelet basis has not been considered in this work but that several optimal selection algorithms have been proposed elsewhere such as best basis [42, ch. 8] or matching pursuit [26], which could be implemented for this purpose. Finally, space limitations prevented presentation of asynchronous multiuser detection results. Similarly to the benefits of using the SAGE algorithm for synchronous multiuser detection reported in [30], the grouped coordinate ascent algorithm can be used to accelerate convergence and to simplify implementation of iterative ML multiuser detection by decoupling the updates of the parameters of each user. Refer to [36] and [37] for details of this extension.

\section{APPENDIX}

The following proposition follows from the definition of $B$ in (22) and assumption i) in that section.

Proposition 1: If $\left|n_{1}-n_{2}\right| \geq 2 B$, then $\nu_{m}^{(i)}\left(n_{1}, n_{2}\right)=0$ for all $m \in \mathbb{Z}$ and index sets $W^{(i)} \subset\{(j, k): j \leq q, k \in \mathbb{Z}\}$.

Proof: The claim will be proved by showing that $d_{j k, m}\left(n_{1}, n_{2}\right)$ are identically zero. In view of (21), it suffices to show that either $c_{j k, m}\left(n_{1}\right)$ or $c_{j k, m}\left(n_{2}\right)$ are identically zero.

Suppose that $n_{2}>n_{1}$ and the pair $(j, k)$ is chosen such that the lower end of the supporting interval of $\psi_{j k}(t)$ overlaps with that of the $n_{1}$ th symbol, i.e.,

$$
2^{j} k-2^{j-1} T_{\psi} \leq n_{1} T_{b}+\frac{T_{p}+T_{0}}{2} .
$$

This implies that $w_{j k}\left(n_{1} ; t\right)$ is possibly nonzero; therefore, its Fourier coefficients $c_{j k, m}\left(n_{1}\right)$ are not identically zero. Now, consider the right endpoint of the supporting interval of $\psi_{j k}(t)$

$$
\begin{aligned}
2^{j} k+2^{j-1} T_{\psi} \leq & n_{1} T_{b}+\frac{T_{p}+T_{0}}{2}+2 \cdot 2^{j-1} T_{\psi} \\
\leq & n_{1} T_{b}+\frac{T_{p}+T_{0}}{2}+2^{q} T_{\psi} \\
\leq & n_{2} T_{b}-2 B T_{b}+\frac{T_{p}+T_{0}}{2}+2^{q} T_{\psi} \\
\leq & n_{2} T_{b}-2\left(\frac{T_{p}+T_{0}}{2}+2^{q-1} T_{\psi}\right) \\
& +\frac{T_{p}+T_{0}}{2}+2^{q} T_{\psi} \\
\leq & n_{2} T_{b}-\frac{T_{p}+T_{0}}{2}
\end{aligned}
$$

The third inequality above follows from the assumption $n_{1} \leq$ $n_{2}-2 B$, and the fourth one follows from (22). This implies that $w_{j k}\left(n_{2} ; d\right)$ is identically zero because $\psi_{j k}(t)$ and $p\left(t-n_{2} T_{b}-d\right)$ have nonoverlapping supporting intervals. Therefore, $c_{j k, m}\left(n_{2}\right)$ are identically zero, and it follows that $d_{j k, m}\left(n_{1}, n_{2}\right)$ are also zero as claimed. A similar proof applies to the case where $n_{1}>n_{2}$.

Next, we show that $\nu_{m}^{(i)}\left(n_{1}, n_{2}\right)$ is zero if the most recent sample in the wavelet index set $W^{(i)}$ is localized at the $n$th symbol, and $n$ is sufficiently smaller than either $n_{1}$ or $n_{2}$. For a set of wavelet indices $W^{(i)}$, recall the definitions of $n_{-}^{(i)}$ and $n_{+}^{(i)}$ in (23).

Proposition 2: If $n_{1} \geq n_{-}^{(i)}+B$ or $n_{2} \geq n_{-}^{(i)}+B$, then $\nu_{m}^{(i)}\left(n_{1}, n_{2}\right)=0$ for all $m \in \mathbb{Z}$. If $n \geq n_{-}^{(\bar{i})}+B$, then $\mu_{m}^{(i)}(n)=0$ for all $m \in \mathbb{Z}$. 
Proof: To show the first part, assume $n_{1} \geq n_{-}^{(i)}+B$; then, for all $(j, k) \in W^{(i)}$

$$
\begin{aligned}
2^{j} k+2^{j-1} T_{\psi} & \leq 2^{j} k+2^{q-1} T_{\psi} \\
& \leq n_{-}^{(i)} T_{b}+2^{q-1} T_{\psi} \\
& \leq\left(n_{1}-B\right) T_{b}+2^{q-1} T_{\psi} \\
& \leq n_{1} T_{b}-\frac{T_{p}+T_{0}}{2}
\end{aligned}
$$

where the second inequality follows from the definition of $n_{-}^{(i)}$ and the third from the assumption on $n_{1}$. It follows that $c_{j k, m}\left(n_{1}\right)=0$ for all $m \in \mathbb{Z}$, which implies $\nu_{m}^{(i)}\left(n_{1}, n_{2}\right)=$ 0 . The rest of the claim follows similarly.

The next proposition states conditions under which $\mu_{m}^{(i)}(n)$ and $\nu_{m}^{(i)}\left(n_{1}, n_{2}\right)$ do not change for an increasing sequence of wavelet index sets $W^{(i)}$.

Proposition 3: If $n_{1} \leq n_{+}^{(i)}-B$ or $n_{2} \leq n_{+}^{(i)}-B$ for some index set $W^{(i)}$, then $\nu_{m}^{(p)}\left(n_{1}, n_{2}\right)=\nu_{m}^{(i)}\left(n_{1}, n_{2}\right)$ for all $p \geq i$, and $m \in \mathbb{Z}$. If $n \leq n_{+}^{(i)}-B$ for some wavelet index set $W^{(i)}$, then $\mu_{m}^{(p)}(n)=\mu_{m}^{(i)}(n)$ for all $p \geq i$ and $m \in \mathbb{Z}$.

Proof: From (21), we have

$$
\nu_{m}^{(p)}\left(n_{1}, n_{2}\right)=\nu_{m}^{(i)}\left(n_{1}, n_{2}\right)+\sum_{j k \in W^{(p)}-W^{(i)}} d_{j k, m}\left(n_{1}, n_{2}\right) .
$$

Consider $d_{j k, m}\left(n_{1}, n_{2}\right)$ for all $(j, k) \in W^{(p)}-W^{(i)}$, and assume that $p>i$ and that the difference set is not empty. Then, the left endpoint of the supporting interval of $\psi_{j k}(t)$ satisfies

$$
\begin{aligned}
2^{j} k-2^{j-1} T_{\psi} & \geq 2^{j} k-2^{q-1} T_{\psi} \\
& \geq n_{+}^{(i)} T_{b}-2^{q-1} T_{\psi} \\
& \geq n_{1} T_{b}+B T_{b}-2^{q-1} T_{\psi} \\
& \geq n_{1} T_{b}+\frac{T_{p}+T_{0}}{2}
\end{aligned}
$$

where the second inequality follows from the definition of $n_{+}^{(i)}$ and assumption iii) and the third from the assumption on $n_{1}$. The last inequality shows that the right endpoint of $p\left(t-n_{1} T_{b}-d\right)$ is smaller than or equal to the left endpoint of $\psi_{j k}(t)$ whenever $|d| \leq T_{0} / 2$. Consequently, $c_{j k, m}\left(n_{1}\right)$ is zero for all $m \in \mathbb{Z}$, which implies that $d_{j k, m}\left(n_{1}, n_{2}\right)$ is zero for $(j, k) \in W^{(p)}-W^{(i)}$. This proves the claim. The proofs for the other cases are similar.

\section{REFERENCES}

[1] P. L. Ainsleigh and C. K. Chui, "A B-wavelet-based noise-reduction algorithm," IEEE Trans. Signal Processing, vol. 44, pp. 1279-1284, May 1996.

[2] J. W. M. Bergmans and H. W. Wong-Lam, "A class of data-aided timing-recovery schemes," IEEE Trans. Commun., vol. 43, nos. 2/4, pp. 1819-1827, Feb./Apr. 1995.

[3] C. Bouman and K. Sauer, "A local update strategy for iterative reconstruction from projections," IEEE Trans. Acoust., Speech, Signal Processing, vol. 41, pp. 534-548, Feb. 1993.

[4] _ "A unified approach to statistical tomography using coordinate descent optimization," IEEE Trans. Med. Imag., to be published.

[5] K.-H. Chang and C. N. Georghiades, "Joint maximum-likelihood timing and data estimation for MSK signals from matched-filter samples," in Proc. IEEE ICC, 1994, vol. 3.

[6] I. Daubechies, "Orthonormal bases of compactly supported wavelets," Commun. Pure Appl. Math., vol. 41, no. 7, pp. 909-996, 1988.
[7] _ "The wavelet transform, time-frequency localization and signal analysis," IEEE Trans. Infrm Theory, vol. 36, pp. 961-1005, Sept. 1990.

[8] __ Ten Lectures on Wavelets. Philadelphia, PA: SIAM, 1992.

[9] A. P. Dempster, N. M. Laird, and D. B. Rubin, "Maximum likelihood from incomplete data via the EM algorithm," J. Roy. Stat. Soc. B, vol. 39, no. 1, pp. 1-38, 1977.

[10] D. L. Donoho, "Nonlinear wavelet methods for recovery of signals, densities, and spectra from indirect and noisy data," in Different Perspectives on Wavelets, I. Daubechies, Ed. Providence, RI: Amer. Math. Soc., 1993.

[11] M. Feder and J. A. Catipovic, "Algorithms for joint channel estimation and data recovery-application to equalization in underwater communications," IEEE J. Oceanic Eng., vol. 16, pp. 42-55, Jan. 1991.

[12] M. Feder and E. Weinstein, "Parameter estimation of superimposed signals using the EM algorithm," IEEE Trans. Acoust., Speech, Signal Processing, vol. 36, pp. 477-489, Apr. 1988.

[13] J. A. Fessler and A. O. Hero, "Space-alternating generalized expectationmaximization algorithm," IEEE Trans. Acoust., Speech, Signal Processing, vol. 42, pp. 2664-2677, Oct. 1994.

[14] _ "Penalized maximum-likelihood image reconstruction using space-alternating generalized EM algorithms," IEEE Trans. Image Processing, vol. 4, pp. 1417-1429, Oct. 1995.

[15] G. D. Forney, "Maximum-likelihood sequence estimation of digital sequences in the presence of intersymbol interference," IEEE Trans. Inform. Theory, vol. IT-18, pp. 363-378, May 1972.

[16] C. N. Georghiades and D. L. Snyder, "The expectation-maximization algorithm for symbol unsynchronized sequence detection," IEEE Trans. Commun, vol. 39, pp. 54-61, Jan. 1991.

[17] C. N. Georghiades and J. C. Han, "Sequence estimation in the presence of random parameters via the EM algorithm," IEEE Trans. Commun., vol. 45, pp. 300-308, Mar. 1997.

[18] G. H. Golub and C. F. Van Loan, Matrix Computations. Baltimore, MD: Johns Hopkins Univ. Press, 1983.

[19] J. Huber and W. Liu, "Data-aided synchronization of coherent CPMreceivers," IEEE Trans. Commun., vol. 40, pp. 178-189, Jan. 1992

[20] _ _ "Data-aided synchronization of M greater than equivalent to 2ary coherent CPM receivers," in Proc. IEEE GLOBECOM, 1990, vol. 2, pp. 709-713.

[21] R. A. Iltis, "An EKF-based joint estimator for interference, multipath, and code delay in a DS spread-spectrum receiver," IEEE Trans. Commun, vol. 42, pp. 1288-1299, Feb./Apr. 1994.

[22] H. Kobayashi, "Simultaneous adaptive estimation and decision algorithm for carrier modulated data transmission systems," IEEE Trans. Commun., vol. COMM-19, pp. 268-280, June 1971.

[23] W. C. Lindsey and M. K. Simon, "Data-aided carrier tracking loops," IEEE Trans. Commun, vol. COMM-19, pp. 157-168, Apr. 1971.

[24] _ Telecommunication Systems Engineering. Englewood Cliffs, NJ: Prentice-Hall, 1973

[25] S. G. Mallat, "Multiresolution approximations and wavelet orthonormal bases of $L^{2}(\mathbb{R}), "$ Trans. Amer. Math. Soc., vol. 315, no. 1, pp. 69-87, 1989.

[26] S. G. Mallat and Z. Zhang, "Matching pursuits with time-frequency dictionaries," IEEE Trans. Signal Processing, vol. 41, pp. 3397-3415, Dec. 1993.

[27] M. H. Meyers and L. E. Franks, "Joint carrier phase and symbol timing recovery for PAM systems," IEEE Trans. Commun., vol. COMM-28, pp. 1121-1129, Aug. 1980.

[28] H. Meyr, M. Oerder, and A. Polydoros, "On sampling rate, analog prefiltering, and sufficient statistics for digital receivers," IEEE Trans. Commun., vol. 42, pp. 3208-3214, Dec. 1994.

[29] J. W. Modestino, D. Sargrad, and R. E. Bollen, "Use of coding to combat impulse noise on digital subscriber loops," IEEE Trans. Commun., vol. 36, pp. 529-537, May 1988 .

[30] L. B. Nelson and H. V. Poor, "Iterative multiuser receivers for CDMA channels: An EM-based approach," IEEE Trans. Commun., vol. 44, pp. 1700-1710, Dec. 1996.

[31] A. V. Oppenheim and R. W. Schafer, Discrete-Time Signal Processing. Englewood Cliffs, NJ: Prentice-Hall, 1989.

[32] J. Qin, "Demodulation of binary PSK signals without explicit carrier synchronization," in Proc. 1993 IEEE Int. Conf. Commun., 1993, pp. 498-501.

[33] S.-C. Rezeanu, R. E. Ziemer, and M. A. Wickert, "Joint maximumlikelihood parameter estimation for burst DS spread-spectrum transmission," IEEE Trans. Commun., vol. 45, pp. 227-238, Feb. 1997.

[34] S.-C. Rezeanu and R. E. Ziemer, "Joint maximum-likelihood data and bit synchronization epoch estimation for burst direct sequence spread-spectrum transmission," Proc. IEEE MILCOM, 1996, vol. 3, pp. 801-805. 
[35] O. Rioul and P. Duhamel, "Fast algorithms for discrete and continuous wavelet transforms," IEEE Trans. Inform. Theory, vol. 38, pp. 569-586, Mar. 1992.

[36] I. Sharfer, "Recursive algorithms for digital communication using the discrete wavelet transform," Ph.D. dissertation, Univ. Michigan, Ann Arbor, 1996.

[37] I. Sharfer and A. O. Hero, "Iterative maximum likelihood sequence estimation for CDMA systems using grouped ascent and the DWT," in Proc. 1997 IEEE Workshop Signal Process. Adv. Commun., Paris, France, Apr. 1997, pp. 137-140.

[38] D. Slepian, "On bandwidth," Proc. IEEE, vol. 64, pp. 292-300, Mar. 1976.

[39] M. A. Tanner, Tools for Statistical Inference: Methods for the Exploration of Posterior Distributions and Likelihood Functions. New York: Springer-Verlag, 1993.

[40] J. R. Thompson and R. A. Tapia, Nonparametric Function Estimation, Modeling, and Simulation. Philadelphia, PA: SIAM, 1990.

[41] M. Vetterli and J. Kovacevic, Wavelets and Sub-Band Coding. Englewood Cliffs, NJ: Prentice-Hall, 1995.

[42] M. V. Wickerhauser, Adapted Wavelet Analysis from Theory to Software. Wellesley, MA: Peters, 1994.

[43] G. W. Wornell, "Emerging applications of multirate signal processing and wavelets in digital communications," Proc. IEEE, vol. 84, pp. 586-603, Apr. 1996.

[44] K. M. Wong, J. Wu, T. N. Davidson, and Q. Jin, "Wavelet packet division multiplexing and wavelet packet design under timing error effects," IEEE Trans. Signal Processing, to be published.

[45] J. Wu, Q. Jin, and K. M. Wong, "Multiplexing based on wavelet packets," in Wavelet Applications II, H. H. Szu, Ed., Proc. SPIE, pp. 315-326 1995.

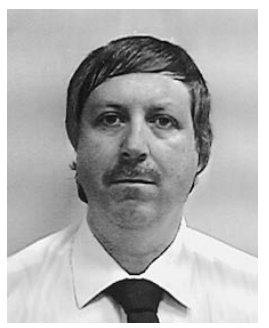

Ilan Sharfer (M'96) was born in Tel-Aviv, Israel, on October 24, 1955. He received the B.Sc. degree (with honors) in electronic and computer engineering from the Technion-Israel Institue of Tecnology, Haifa, in 1977, the M.Sc. degree (with honors) from Tel-Aviv University in 1991, and the Ph.D. degree from the University of Michigan, Ann Arbor, in 1996, both in electrical engineering.

From 1981 to 1982 , he studied for the Master's degree in electronic engineering at the Philips International Institute (PII), Eindhoven, The Netherlands. From 1983 to 1992, he worked at National Semiconductor, Herzlia, Israel, as a VLSI design engineer. Since 1998, he has been with Orckit Communications, Tel-Aviv, developing DSP algorithms in the area of digital communications. $\mathrm{He}$ is also a Lecturer on signal processing in the Communications Systems Engineering Department, Ben-Gurion University, Beer-Sheva, Israel. His research activities include the areas of signal processing and statistical communications theory, specifically higher order spectra, applications of wavelet theory in digital communications, channel estimation, and synchronization algorithms.

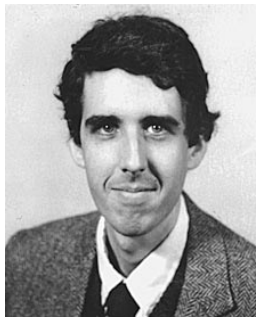

Alfred O. Hero, III (S'79-M'84-SM'96-F'97) was born in Boston, MA, in 1955. He received the B.S. degree (summa cum laude) from Boston University in 1980, and the Ph.D. degree from Princeton University, Princeton, NJ, in 1984, both in electrical engineering. He held the honorary G. V. N. Lothrop Fellowship in Engineering at Princeton University.

Since 1984, he has been with the Departmen of Electrical Engineering and Computer Science, University of Michigan, Ann Arbor, where he is currently Professor and Director of the Communications and Signal Processing Laboratory. He has held the positions of Visiting Scientist at the Massachusetts Institute of Technology Lincoln Laboratory, Lexington, from 1987 to 1989; Visiting Professor at l'Ecole Nationale de Techniques Avancees (ENSTA), Paris, France, in 1991; and William Clay Ford Fellow at the Ford Motor Company, Dearborn, MI, in 1993. He has served as consultant for U.S. government agencies and private industry. His present research interests are in the areas of detection and estimation theory, statistical signal and image processing, statistical pattern recognition, signal processing for communications, channel equalization and interference mitigation, spatio-temporal sonar and radar processing, and biomedical signal and image analysis.

Dr. Hero is a member of Tau Beta Pi, the American Statistical Association, the New York Academy of Science, and Commission C of the International Union of Radio Science (URSI). He was Associate Editor for Signal Processing at the IEEE TRANSACTIONS ON INFORMATION THEORY from 1994 to 1997; Chair of the IEEE SPS Statistical Signal and Array Processing Technical Committee from 1996 to 1998; and, since 1997, has been Treasurer of the IEEE SPS Conference Board. He is Cochair for the 1999 IEEE Information Theory Workshop and the 1999 IEEE Workshop on Higher Order Statistics. He served as Publicity Chair for the 1986 IEEE International Symposium on Information Theory and was General Chair of the 1995 IEEE International Conference on Acoustics, Speech, and Signal Processing. 